\title{
Increase the Performance Level of Young Basketball Players through the Use of High Intensity Interval Training
}

\author{
Francesca D’Elia, Danilo D’Andrea, Giovanni Esposito*, Gaetano Altavilla, Gaetano Raiola \\ Department of Political and Social Studies, University of Salerno, Salerno, 84084, Fisciano, Italy
}

Received January 30, 2021; Revised March 22, 2021; Accepted April 18, 2021

\section{Cite This Paper in the following Citation Styles}

(a): [1] Francesca D’Elia, Danilo D’Andrea, Giovanni Esposito, Gaetano Altavilla, Gaetano Raiola , "Increase the Performance Level of Young Basketball Players through the Use of High Intensity Interval Training," International Journal of Human Movement and Sports Sciences, Vol. 9, No. 3, pp. 445 - 450, 2021. DOI: 10.13189/saj.2021.090308.

(b): Francesca D'Elia, Danilo D'Andrea, Giovanni Esposito, Gaetano Altavilla, Gaetano Raiola (2021). Increase the Performance Level of Young Basketball Players through the Use of High Intensity Interval Training. International Journal of Human Movement and Sports Sciences, 9(3), 445 - 450. DOI: 10.13189/saj.2021.090308.

Copyright@2021 by authors, all rights reserved. Authors agree that this article remains permanently open access under the terms of the Creative Commons Attribution License 4.0 International License

\begin{abstract}
The aim of this study is to investigate the effectiveness of a specific 12-week HIIT methodology for improving aerobic and anaerobic performance in a group of young basketball players. Twenty-two $(\mathrm{n}=22)$ players participated in the study. (age 19.1 \pm 1.1 ; height: $185 \pm 5.2$; body mass: $70.9 \pm 6.0$ ). In the pre-season (4 weeks, from July to August), the players performed Pizzolato's 7-minutes test, to evaluate aerobic and anaerobic performance before the start of the season. After 12 weeks of training (in-season), using the Tabata training methodology, the same players repeated Pizzolato's 7-minutes test to look for improvements in performance and evaluate if the distance covered during the test is better, equal, or worse compared to the pre-season. After 12 weeks of training, in the experimental group we found a significant increase in the values of $\mathrm{VO}_{2} \max (p=0.02)$ and SAN ( $=0.01)$ and a significant decrease in the values of HRmax ( $p=0.00)$, such as to be able to attribute them, with a margin of error of $5 \%$, to the benefits produced by a twelve-week mesocycle based on HIIT principles. No differences between groups were found in the control group. The effectiveness of specific High Intensity Interval Training has been revealed in improving aerobic performance in young basketball players.
\end{abstract}

Keywords Aerobic Fitness, Field Tests, Young Players, High Intensity Interval Training

\section{Introduction}

The modern basketball player must possess special physical qualities for effective game development. A characteristic of this sport is the continuous repetition of such efforts, under the pressure of one or more opponents, and with very short recovery times [5, 31]. From a physiological-biomechanical point of view, basketball is classified among the sports activities with alternating aerobic-anaerobic energy commitment, with district demands of medium to high strength and endurance. Specific technical, coordinative, and psychological qualities are also important [27]. In basketball, moments of mainly aerobic commitment (running at low speed, walking, recoveries, and game breaks) alternate with others with anaerobic commitment of the alactic type (running strokes, jumps, sprints, accelerations, changes of direction and speed). Perhaps, even a lactic component in the performance should not be overlooked [2, 4]. Like all team sports, it too requires the realization of a series of intermittent efforts, or the alternation of short sprints and jumps with active or passive pauses [25]. It is characterized by a heart rate (HR) that has a range between $80 \%$ and $95 \%$ of HRmax and a percentage of $\mathrm{VO}_{2}$ max between $70 \%$ and $80 \%$. Finally, the game/pause ratio is $1: 1$ and, more frequently, the actions take place around $20-40$ seconds [12, 18].

Taking this information into account, the main goal of 
the coach in planning and periodizing training must be to obtain the maximum athletic form in his players. The "peak form" represents the synthesis, at the highest level, of the motor, energetic, technical, and psychological potentialities that an athlete can reach based on his starting level [13]. According to Bompa [10], peak conditioning is essential to achieving optimal athletic performance. The physical demands of different sports vary significantly. Therefore, the athlete who wishes to specialize or excel in a single sport should follow a sport-specific exercise prescription. Among the various valid training methodologies present in the literature, this study focuses its attention on the potential of High Intensity Interval Training (HIIT) for the need to modify some physiological characteristics in athletes. HIIT is an advanced form of Interval Training that involves alternating periods of short and intense anaerobic exercise and less intense periods of active recovery. It also consists in the variation of the heart rate through a continuous passage from moderate to high frequencies and vice versa during the same exercise [7]. This type of recovery favours high intensity anaerobic performance because it allows to dispose of part of the accumulated lactate. It is a form of mixed cardiovascular training compared to the more popular moderate intensity, constant heart rate aerobic training, Steady State Training (SST). It is useful as a strategy for weight loss and the improvement of cardiovascular capacity [3]. The average time it takes to complete a work session can be around 20 minutes or less and can help improve cardiovascular capacity and reduce body fat like or more than classic moderate-intensity aerobic activity [19]. The intensities usually used in the active recovery phase amount to about $60 \%$, while the peak intensities reach average levels between 85 and $90 \%$ of maximum heart rate (HRmax). The intensity parameter can be measured through different methods, and in scientific research the percentage of maximum oxygen consumption $\left(\mathrm{VO}_{2} \mathrm{max}\right)$ is more commonly used to establish it [20,23]. One of the most interesting aspects is that High Intensity Interval Training can produce the same physiological benefits as Steady State Training, even if the duration of the session is more than half less. Recent studies have shown the positive impact of High intensity Interval Training on the aerobic performance of team sports athletes. Delextrat et al. [14], found a similar increase in maximum aerobic performance among teenage basketball players after adding 12 HIIT sessions to training during the season. The study by Eather, et al. [15], showed significant improvements in aerobic performance after implementing specific high-intensity training with various changes of direction in basketball players. From the literature review it is possible to identify the main problem of our research: there are several training methodologies that fall under the HIIT method, but only some of them have been tested as efficient in basketball, unlike how it happens in other team sports. The purpose of this study is to demonstrate that introducing a specific HIIT training methodology into the training practice of amateur basketball players will help improve both aerobic power and anaerobic capacity at the same time.

\section{Materials and Methods}

\subsection{Participants}

Twenty-two ( $\mathrm{n}=22)$ young amateur basketball players participated in this study (age: $19.1 \pm 1.1$; height: $185 \pm 5.2$; body mass: $70.9 \pm 6.0$; fat mass: $18.1 \pm 4.8$ ). To be included in the study, subjects had to 1) ensure regular participation in all the training sessions, 2) have competed regularly during the previous competitive season, and 3) possess medical clearance. Prior to joining the study, participants were briefed on the study objectives and procedures and provided written informed consent prior to the testing procedure. The study protocol complied with the World Medical Association's code of ethics (Declaration of Helsinki). The basketball team trained for about 1 hour three times a week (always Monday, Wednesday, and Friday) plus the official match played on Saturday or Sunday. The study was conducted during the 2019-2020 competitive season (from July to October).

\subsection{Study Design}

The study was conducted during a mesocycle of the competitive season. It included three weekly training sessions (each lasting two hours) for a total duration of twelve weeks. In addition to team training, the players played one match per week. Eleven players (experimental group) integrated a basketball specific HIIT program into their weekly training, while the other 11 players served as a control group and continued their traditional training routine. The players were distributed in each group evenly.

The workouts began with a specific twenty-minute warm-up. Subsequently, the experimental group had to perform specific high-intensity training, while the control group conducted an intensive transition exercises or a specific situational game. This differentiated part of the training was then followed by technical and tactical exercises and a final match at the end of each session, identical for both groups. Overall, both groups spent the same amount of time on the training practice. In a first phase of evaluation, before carrying out the differentiated training mesocycle, the initial values of $\mathrm{VO}_{2} \max$ and anaerobic threshold (SAN) were measured in all the players by means of the Pizzolato's 7-minutes test. Péronnet has shown that this test is based on this specific time lapse because, close to the maximum oxygen consumption, an athlete is able to maintain the effort for about seven minutes [28]. Therefore, the maximum aerobic speed can be kept for a period between 5 and 9 
minutes and the $\mathrm{VO}_{2}$ max value can be extrapolated from this. This test allows to indirectly measure the values of $\mathrm{VO}_{2}$ max and SAN, detecting the maximum distance that an athlete can cover in seven minutes, at maximum speed. The test can be considered reliable if the speed is kept constant for the duration of the effort. Tanaka's formula was then used to calculate HRmax. It consisted in applying the following formula: 208 - (product between 0.7 and the age expressed in years). Following the application of the test (pre-test), the players carried out a twelve-weeks differentiated training mesocycle. Among the various HIIT training methodologies, the preparation mesocycle, which the control group performed, was based on the Tabata protocol, which proved to be very effective in several team sports [16, 22]. This protocol was created with the aim of improving the aerobic and anaerobic characteristics beyond the threshold (anaerobic). It consists of performing seven or eight repetitions at maximum intensity, alternating with ten seconds of passive recovery. The series lasts approximately four minutes $(240 \mathrm{sec}$ ). Requires an intensity of approximately $170 \%$ of $\mathrm{VO}_{2}$ max for the duration of the session. Given the intensity of the Tabata protocol, it is necessary to finish the workout with a cool down of at least 10-15 minutes. The main feature of the Tabata protocol is also to increase the heart rate (HR) until reaching the maximum sustainable levels, making full use of both aerobic and anaerobic metabolism [21]. Through short breaks, which allow to recover and, only partially, to dispose of the lactic acid produced and accumulated, the Tabata protocol allows to remain at the highest levels of intensity, or almost, for a long period of time, up to performing the eight repetitions. In a short time, the Tabata protocol induces a high oxygen debt that lasts until the end of the workout. It includes exercises like push-ups, split squats, box jumps, burpees, jumping rope, jumping jacks and more (figure 1).

\begin{tabular}{|l|c|c|c|c|} 
& Minute 1 & Minute 2 & Minute 3 & Minute 4 \\
\hline Segment 1 & High Knee Run & Plank Punch & Jumping Jacks & Side Skaters \\
\hline Segment 2 & Jump Rope & In/Out Boat & Line Jumps & Push-Ups \\
\hline Segment 3 & Burpees & Russian Twists & Squats & Lunges \\
\hline Segment 4 & Mt. Climbers & Push-Ups & Split Squat & Box Jump \\
\hline
\end{tabular}

Figure 1. Tabata workout Protocol

This training program, lasting about 20 minutes, was repeated three times a week for a total of 12 weeks. The duration of this type of work is not accidental. In fact, it has been scientifically established that only after this period it is possible to find significant improvements in $\mathrm{VO}_{2} \max$, HRmax and anaerobic threshold. At the end of the twelve weeks of training, Pizzolato's 7-minutes test was performed again to measure $\mathrm{VO}_{2} \max$ and $\mathrm{SAN}$ values. Tanaka formula was used to derive the HRmax.

\subsection{Statistical Analysis}

The results are presented as the mean and standard deviation. The normality of the distribution with the Shapiro Wilk test was determined. Paired difference t-test was used to combine the results obtained from the tests before the start of the specific training mesocycle, and at the end of it, after twelve weeks. The analyses were performed with $95 \%$ confidence interval and $p \leq 0.05$. The statistical analyses were performed with SPSS Statistics version 23.

\section{Results}

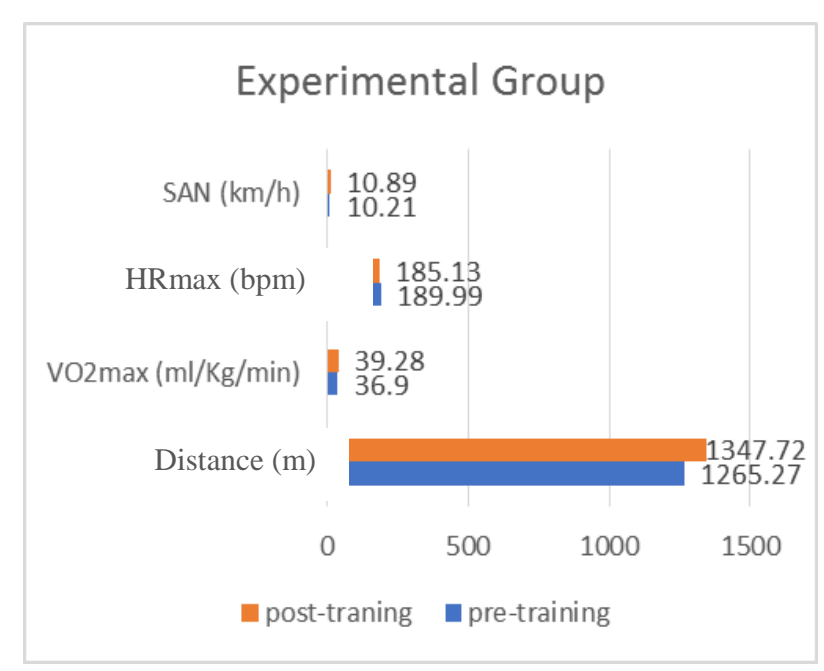

Figure 2. Results of Experimental group

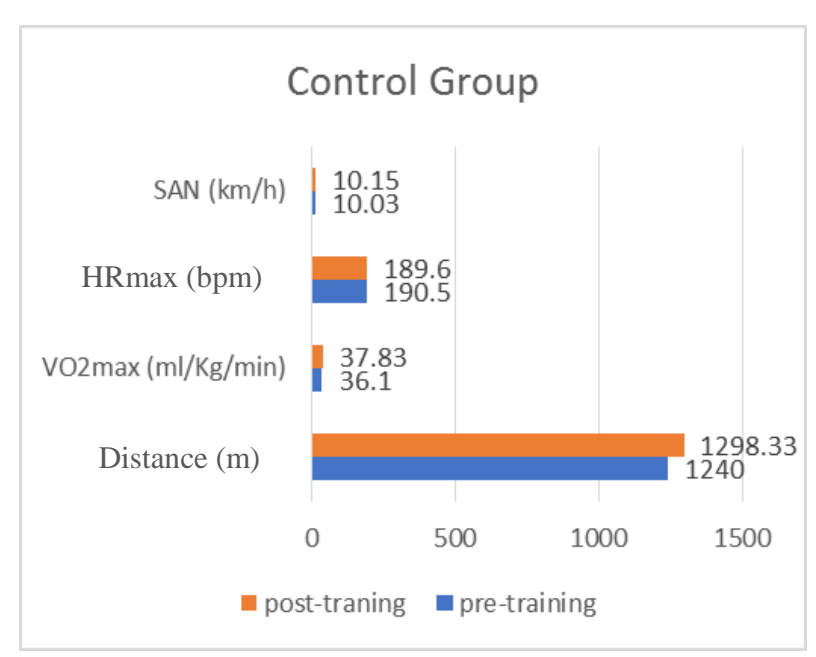

Figure 3. Results of Control Group

Table 1 shows the mean and SD of the distance traveled (m) in the Pizzolato's 7-minutes test performed in the pre-season, and the values of the $\mathrm{VO}_{2} \max$, HRmax and Anaerobic Threshold (SAN) for the experimental and control group. Table 2 shows the same results performed after the 12-week differentiated specific training 
(in-season). Table 3 and Table 4 show the statistical analyzes of the independent and dependent variables, respectively, obtained using a paired t-test for the experimental and control group. From the data analysis it emerged that the experimental group recorded an extremely significant correlation in the parameters of distance, $\mathrm{VO}_{2} \max , \mathrm{HRmax}$ and SAN (figure 2). This allows to establish, according to what is the objective of our study, that the differences between the data acquired before and after the training methodology are so significant that they can be attributed to the methodology itself. In fact, we found an important significance in the increase of $\mathrm{VO}_{2}$ max and SAN values, and in the decrease in HRmax values, such that they can be attributed, with a margin of error of $5 \%$, to the benefits produced by a twelve-week mesocycle based on High Intensity Interval Training's principles. As for the control group, the correlation shows a slight improvement of the three parameters but, in this case, the correlation is not significant (Figure 3).
Table 1. Test results obtained from the experimental group and the control group performed in pre-season.

\begin{tabular}{|c|c|c|c|c|}
\hline \multirow{2}{*}{$\begin{array}{c}\text { Pizzolato's } \\
\text { 7-minutes test }\end{array}$} & \multicolumn{2}{|c|}{ Experimental group } & \multicolumn{2}{c|}{ Control group } \\
\cline { 2 - 5 } & Mean & SD & Mean & SD \\
\hline Distance (m) & 1265.2 & 8.11 & 1240 & 9.07 \\
\hline $\begin{array}{c}\mathrm{VO}_{2} \max \\
(\mathrm{ml} / \mathrm{Kg} / \mathrm{min})\end{array}$ & 36.9 & 1.11 & 35.8 & 1.09 \\
\hline HRmax (bpm) & 189.99 & 3.26 & 190.5 & 2.52 \\
\hline SAN (km/h) & 10.21 & 0.31 & 10.03 & 0.32 \\
\hline
\end{tabular}

Table 2. Test results obtained from the experimental group and the control group performed in-season (after 12 weeks)

\begin{tabular}{|c|c|c|c|c|}
\hline \multirow{2}{*}{$\begin{array}{c}\text { Pizzolato's } \\
\text { 7-minutes test }\end{array}$} & \multicolumn{2}{|c|}{ Experimental group } & \multicolumn{2}{c|}{ Control group } \\
\cline { 2 - 5 } & Mean & SD & Mean & SD \\
\hline Distance (m) & 1347.7 & 9.15 & 1257.3 & 8.23 \\
\hline $\begin{array}{c}\mathrm{VO}_{2} \mathrm{max} \\
(\mathrm{ml} / \mathrm{Kg} / \mathrm{min})\end{array}$ & 39.2 & 2.81 & 36.2 & 1.87 \\
\hline $\mathrm{HRmax}(\mathrm{bpm})$ & 185.1 & 4.07 & 189.9 & 3.60 \\
\hline $\mathrm{SAN}(\mathrm{km} / \mathrm{h})$ & 10.89 & 0.78 & 10.59 & 0.16 \\
\hline
\end{tabular}

Table 3. Results of the statistical analysis based on the use of the paired differences t-test for the data of the experimental group.

\begin{tabular}{|c|c|c|c|c|c|c|c|c|}
\hline & \multicolumn{5}{|c|}{ Paired Differences } & \multirow{3}{*}{$\mathrm{t}$} & \multirow{3}{*}{ df } & \multirow{3}{*}{ Sign. (Two-tailed) } \\
\hline & \multirow{2}{*}{ Mean } & \multirow{2}{*}{ Std. Deviation } & \multirow{2}{*}{ Std. Error Mean } & \multicolumn{2}{|c|}{\begin{tabular}{|c} 
95\% Confidence Interval of \\
the Difference
\end{tabular}} & & & \\
\hline & & & & Lower & Upper & & & \\
\hline $\begin{array}{l}\mathrm{VO}_{2} \text { max pre-season - } \\
\mathrm{VO}_{2} \text { max in-season }\end{array}$ & 38.05 & 1.96 & 3.96 & 1.70 & 2.05 & 3.24 & 10 & 0.02 \\
\hline $\begin{array}{l}\text { Distance pre-season - } \\
\text { Distance in-season }\end{array}$ & 1306.4 & 8.63 & 1,22 & 1.78 & 2.17 & -2.7 & 10 & 0.01 \\
\hline $\begin{array}{l}\text { SAN pre-season - } \\
\text { SAN in-season }\end{array}$ & 10.55 & 0.54 & 0.30 & 1.78 & 2.17 & -2.74 & 10 & 0.01 \\
\hline $\begin{array}{l}\text { HRmax pre-season - } \\
\text { HRmax in-season }\end{array}$ & 187.5 & 3.66 & 12.9 & 1.73 & 2.10 & 3.00 & 10 & 0.00 \\
\hline
\end{tabular}

Table 4. Results of the statistical analysis based on the use of the paired differences t-test for the data of the control group.

\begin{tabular}{|c|c|c|c|c|c|c|c|c|}
\hline & \multicolumn{5}{|c|}{ Paired Differences } & \multirow{3}{*}{$\mathrm{t}$} & \multirow{3}{*}{$\mathrm{df}$} & \multirow{3}{*}{ Sign. (Two-tailed } \\
\hline & \multirow{2}{*}{ Mean } & \multirow{2}{*}{ Std. Deviation } & \multirow{2}{*}{ Std. Error Mean } & \multicolumn{2}{|c|}{\begin{tabular}{|c|}
$\begin{array}{c}95 \% \text { Confidence Interval of } \\
\text { the Difference }\end{array}$ \\
\end{tabular}} & & & \\
\hline & & & & Lower & Upper & & & \\
\hline $\begin{array}{c}\mathrm{VO}_{2} \text { max pre-season - } \\
\mathrm{VO}_{2} \text { max in-season }\end{array}$ & 36.45 & 8.65 & 1.21 & 1.75 & 2.13 & -0.27 & 10 & 0.78 \\
\hline $\begin{array}{l}\text { Distance pre-season - } \\
\text { Distance in-season }\end{array}$ & 1249.8 & 8.12 & 2.34 & 1.81 & 2.22 & -1.54 & 10 & 0.15 \\
\hline $\begin{array}{l}\text { SAN pre-season - } \\
\text { SAN in-season }\end{array}$ & 10.22 & 0.24 & 1.21 & 1.79 & 2.20 & -0.87 & 10 & 0.39 \\
\hline $\begin{array}{l}\text { HRmax pre-season - } \\
\text { HRmax in-season }\end{array}$ & 190.05 & 3.06 & 1.34 & 0.26 & 0.52 & 0.64 & 10 & 0.52 \\
\hline
\end{tabular}




\section{Discussion}

We have shown that these sport-specific HIIT sessions can be applied to increase the aerobic and anaerobic performance of basketball players, with no adverse effects on power-related performance.

Implementing basketball-specific high-intensity training is time-efficient for two reasons. One reason is the shorter time required compared to high-volume resistance training; the other reason is the integration of technical skills into high intensity training [26]. The exercises were carried out at high intensity, allowing each player to rest even for a few seconds. To set up an adequate metabolic stimulus, it is important to exercise $90-95 \%$ of the individual maximum heart rate without recovery within intervals [33]. The exercises conducted by the experimental group were designed to ensure a continuous load for the set time interval (respectively 4 min and 30 s). Based on our results, a basketball specific HIIT, with short and long intervals, can be conducted to improve aerobic performance in young players.

The results of this study are consistent with those of the literature, which state that a well-structured high-intensity training brings significant benefits to the physiological and morpho-functional characteristics of the athlete, in less time than continuous training $[1,9,30]$. The Tabata protocol has been confirmed as an effective workout to simultaneously increase both aerobic power (by increasing the anaerobic threshold) and anaerobic capacity (such as, for example, lactic power and lactate tolerance) [6]. Other studies previously conducted on the Tabata protocol have shown how this methodology can confer non-negligible benefits on the body's physical abilities in just 12 weeks (42 days, or almost 1 month and a half) [24, 29]. The improvements observed concerned both aerobic capacity (with the increase in maximum oxygen consumption), and anaerobic capacity, (increasing the ability to tolerate oxygen debt). These studies show how physical activity at maximal or sub-maximal intensity can improve some components of aerobic capacity more than low and medium intensity aerobic activity itself, although some consider the opposite still valid $[8,17]$. Furthermore, our results are consistent with those of a recent meta-analysis [32], which shows how High Intensity Interval Training can increase $\mathrm{VO}_{2}$ max from $4.2 \%$ to $13.4 \%$. The adaptations are mainly peripheral and concern the oxidative potential of the muscle [11]. Some limitations of this study design must be considered. The first of these relates the small number of participants who took part in the study. The second, on the other hand, concerns the lack of the possibility of using expensive and sophisticated equipment to directly measure the values of $\mathrm{VO}_{2}$ max and SAN. In a functional evaluation conducted in the field, as in our case, indirect tests were used to collect information on some characteristics of the players that cannot be measured directly outside the laboratories. Obviously, these indirect tests could, for example, not ensure the same accuracy in the measurement compared to the analysis of exhaled gases at the end of an incremental test.

Finally, to further highlight in other studies the enormous benefits provided by a HIIT training mesocycle, it would be interesting to be able to measure the values of $\mathrm{VO}_{2}$ max, SAN and HRmax throughout the season. Therefore, the benefits obtained after a high intensity Interval Training macrocycle could be compared with those of the mesocycle measured in this study. In fact, apart from the knowledge of the effectiveness of HIIT in improving the fitness of athletes, little is known about the long-term impact on potential training adaptations following the implementation of HIIT in basketball practice.

\section{Conclusions}

Our results suggest that through a twelve-weeks training mesocycle based on the High Intensity Interval Training's principles, we can positively condition the physical and physiological development of our players, in such a way as to provide them with the opportunity to express the highest level of performance in the game of basketball. Therefore, it is possible to train players with HIIT sessions, preceded by a Steady State Training mesocycle, to obtain much more significant results in less time.

\section{REFERENCES}

[1] Abdelkrim NB, El Fazaa S, El Ati J. Time-motion analysis and physiological data of elite under-19-year-old basketball players during competition. British journal of sports medicine 2007; 41(2): 69-75.

[2] Abdelkrim NB, Castagna C, El Fazaa S, El Ati J. The effect of players' standard and tactical strategy on game demands in men's basketball. The Journal of Strength \& Conditioning Research 2010; 24(10): 2652-2662.

[3] Alonso-Fernández D, Fernández-Rodríguez R, Taboada-Iglesias Y, Gutiérrez-Sánchez Á. Impact of a HIIT protocol on body composition and $\mathrm{VO}_{2} \mathrm{max}$ in adolescents. Science \& Sports 2019; 34(5): 341-347.

[4] Altavilla G, Raiola G. Global vision to understand the game situations in modern basketball, Journal of Physical Education and Sport 2014; 14(4): 493-496.

[5] Altavilla G, Raiola G. Sports game tactic in basketball. Sport Science 2015; 8(1): 43-46.

[6] Altavilla G, D'Isanto T, Di Tore PA. Anthropometrics characteristics and jumping ability in basketball. Journal of Human Sport \& Exercise 2018, 13(2): 385-392.

[7] Altinkok M. An analysis on the spheres of influence of high-intensity interval training (HIIT) practices. International Journal of Social Sciences and Education Research 2015; 1(2): 463-475. 
[8] Aryanto B, Sukoco P, Lumintuarso R.The Validity of Construct Analysis on Assessment Instrument of Basketball Skill for Senior High School in Yogyakarta City. International Journal of Human Movement and Sports Sciences 2020; 8(5): $193 \quad-198 . \quad$ DOI: 10.13189/saj.2020.080506.

[9] Aschendorf PF, Zinner C, Delextrat A, Engelmeyer E, Mester J. Effects of basketball-specific high-intensity interval training on aerobic performance and physical capacities in youth female basketball players. The Physician and Sportsmedicine 2019; 47(1): 65-70.

[10]Bompa TO, Haff GG. Periodization. Theory and Methodology of Training. [5-th Edition]. Champaign, IL, USA: Human Kinetics; 2009.

[11] Castagna C, Impellizzeri FM, Rampini E, D'Ottavio S, Manzi $\mathrm{V}$. The Yo-Yo intermittent recovery test in basketball players, Journal of Science and Medicine in Sport 2008; 11: 202-208.

[12] Czuba M, Zając A, Maszczyk A, Roczniok R, Poprzęcki S, Garbaciak W, Zając T. The effects of high intensity interval training in normobaric hypoxia on aerobic capacity in basketball players. Journal of human kinetics 2013; 39(1):103-114

[13] D’Elia F, Rago V, Ermidis G, Raiola G. Relationship between lower limb asymmetries and functional capacities in women in Basketball: A case study. Sport Science 2020; 13 (1): 90-95.

[14] Delextrat A, Gruet M, Bieuzen F. Effects of small-sided games and high-intensity interval training on aerobic and repeated sprint performance and peripheral muscle oxygenation changes in elite junior basketball players. The Journal of Strength \& Conditioning Research 2018; 32(7): 1882-1891.

[15] Eather N, Babic M, Riley N, Harris N, Jung M, Jeff M, Lubans DR. Integrating high - intensity interval training into the workplace: The Work - HIIT pilot RCT. Scandinavian Journal of Medicine \& Science in Sports 2020. https://doi.org/10.1111/sms.13811.

[16] Emberts T, Porcari J, Dobers-tein S, Steffen J, Foster C. Exercise intensity and energy expenditure of a tabata workout. Journal of sports science \& medicine 2013; 12(3): 612.

[17] Espinosa-Mendez CM, Renero-Carrillo FJ, San Martin-Rodriguez SL, Flores-Chico B. The Effect of Respiratory Muscle Training on the Maximum Oxygen Consumption and the Anaerobic Threshold. Sport Mont 2020; 18(3): 57-60.

[18] Falcone G, Scurati R, D'Elia F, D'Isanto T. Basketball and ankle injuries. Journal of Human Sport and Exercise 2019:14 (4):1142-1148.

[19] Grimal Y, Lorenzo A. The effects of including changes of direction (COD) in the high intensity interval training (HIIT) on heart rate and the rate of perceived exertion in young basketball players. Revista de Psicologia del Deporte 2018; 27(2): 165-172.

[20] Keating CJ, Montilla JÁP, Román PÁL, del Castillo RM. Comparison of High-Intensity Interval Training to
Moderate-Intensity Continuous Training in Older Adults: A Systematic Review. Journal of Aging and Physical Activity 2020; 1(aop):1-10.

[21] Limyati Y, Budiman I, Harja RS, Ibrahim I. The Effect of Acute High Intensity Interval Training on Alertness and Executive Function in Basketball Player. Journal of Medicine and Health 2020; 2(6).

[22] Marić B, Marić D. The effect of interval (HIIT) aerobic training on acute muscle damage in woman basketball players related to continuous aerobe training. Glasnik Antropološkog društva Srbije 2018; (53): 21-28.

[23] Mokhtar M, Mohammed Z, Mohamed HK, Wahib B. Maximal Oxygen Levels as an Incremental Exercise to Optimise Individual Training Prescription with the Aim of Rectifying Weaknesses of Elite Algerian Soccer. International Journal of Human Movement and Sports Sciences 2019; 7(4): 74 - 80. DOI: 10.13189/saj.2019.070402.

[24] Nanda FA, Dimyati D. The psychological skills of basketball athletes: Are there any differences based on the playing position?. Jurnal Keolahragaan 2019; 7(1): 74-82.

[25] Padulo J, Bragazzi NL, Nikolaidis PT, Dello Iacono A, Attene G, Pizzolato F, Migliaccio GM. Repeated sprint ability in young basketball players: multi-direction vs. one-change of direction (Part 1). Frontiers in physiology 2016; 7: 133

[26] Raiola, G, D'Isanto T. Descriptive shot analysis in basketball, Journal of Human Sport and Exercise 2016; 11,1 (Special issue).

[27] Raiola G, Tafuri D, Paloma FG, Lipoma M. Bodily Communication in basketball. Sport Sciences 2015; 8(2): 13-18.

[28] Péronnet, F. Le marathon: équilibre énergétique, alimentation et entraînement du coureur sur route. Décarie, 1991.

[29] Rozi MF. Effect of Exercise Method and Nutritional Status of Ability VO2max on Basketball Players Performance. In: 1st International Conference of Physical Education (ICPE 2019). Atlantis Press; 2020: 234-236.

[30] Schelling X, Torres-Ronda L. Conditioning for basketball: Quality and quantity of training. Strength \& Conditioning Journal 2013; 35(6): 89-94.

[31] Stoll O, Lau A, Stoeber J. Perfectionism, and performance in a new basketball training task: Does striving for perfection enhance or undermine performance? Psychology of sport and Exercise 2008; 9(5): 620-629.

[32] Torres-Ronda L, Ric A, Llabres-Torres I, de las Heras B, del Alcazar XS. Position-dependent cardiovascular response and time-motion analysis during training drills and friendly matches in elite male basketball players. The Journal of Strength \& Conditioning Research 2016; 30(1):60-70.

[33] Türker A, Yüksel O. The effect of functional and supportive classic strength trainings in basketball players on aerobic strength, dynamic balance and body composition. Pedagogy of Physical Culture and Sports 2020; 25(1): 47-57 